\title{
Wood made denser and stronger
}

An improved method for compressing wood substantially increases its strength and stiffness, opening up the possibility of applications in engineering for which natural wood is too weak. SEE LETTER P.224

\section{PETER FRATZL}

$\mathrm{W}$ ood is among the oldest materials used by humans, and is still commonly used for building ${ }^{1}$. Its low density has also made it useful for transport applications such as shipbuilding, but this property is associated with a relatively low strength and stiffness. Scientists have tried to devise processes that make wood denser, to obtain materials suitable for high-strength applications, but with limited success. On page 224, Song et al. ${ }^{2}$ describe a densification method that combines a chemical treatment with high-temperature compression, and which produces an unprecedented increase in stiffness and strength.

The authors' method starts by treating wood blocks with sodium hydroxide and sodium sulfite, a chemical process similar to the method used to pulp wood to make paper. This chemical treatment partially removes lignin and hemicelluloses (Fig. 1). Lignin is a biopolymer that has many functions in plants, such as stabilizing cell walls in wood and retarding attacks on wood cells by parasites and bacteria; hemicelluloses are sugar chains that cover and bind fibrils of cellulose in the cell walls.

Song and colleagues then compress the blocks at temperatures of about $100^{\circ} \mathrm{C}$. This removes most of the pores in the wood, and increases its density from 0.43 grams per cubic centimetre to $1.3 \mathrm{~g} \mathrm{~cm}^{-3}$. The resulting stable material is too dense to float on water, but the authors report that its stiffness and strength have both increased impressively, by a factor of about 11 compared with untreated wood. As the authors point out, previous attempts to densify wood also improved the strength, but by no more than a factor of about three to four ${ }^{3}$. The secret to Song and colleagues' success lies in their combination of chemical treatment and high temperatures during pressing.

Natural wood contains a multitude of parallel, tube-like cells, the walls of which constitute the major part of the material. In most parts of woody stems, the cells have died and left behind their cellulose-rich cell walls. These walls also contain lignin and hemicelluloses, and form hollow wood fibres. The tube-like fibres collapse laterally when compacted, effectively losing their hollow interiors. This increases the amount of material per cross-section of the stem, as evidenced by the increased density reported by Song and colleagues. On its own, this effect would be expected to cause the stiffness and strength of wood to increase in proportion to the increase in density ${ }^{4}$.

However, the authors report that the stiffness increases by a factor of 11 , whereas the density increases by a factor of only 3 . A threefold density increase has been observed in previous work that used hot pressing alone (see ref. 3, for example). It therefore seems likely that the authors' chemical treatment modifies and strengthens the cellulose-based composite that makes up cell walls in wood.

Many cellulose-based materials swell undesirably when they come into contact with water, but Song and colleagues report that water swelling of their densified wood is tolerably small. It remains to be seen whether the partial removal of lignin from the material makes it susceptible to bacterial or fungal attacks.

The densified wood is still lighter than metallic materials, so its stiffness and strength open up the potential for many engineering applications. This raises the question of why trees use a porous material for their trunks, when their goal in a forest is to be as high as possible, to ensure that their leaves are exposed to light - a task for which stiffer and stronger materials might intuitively seem better suited. By making wood porous, trees partially sacrifice the material's strength. One answer is that wood is multifunctional, and so the pores are needed for more than just structural tasks, such as to transport water and nutrients.

But the optimal response of natural materials to a load varies according to the function involved, such that lower density can be more important than higher strength ${ }^{5-7}$. In brief, the height of a slender column that supports a
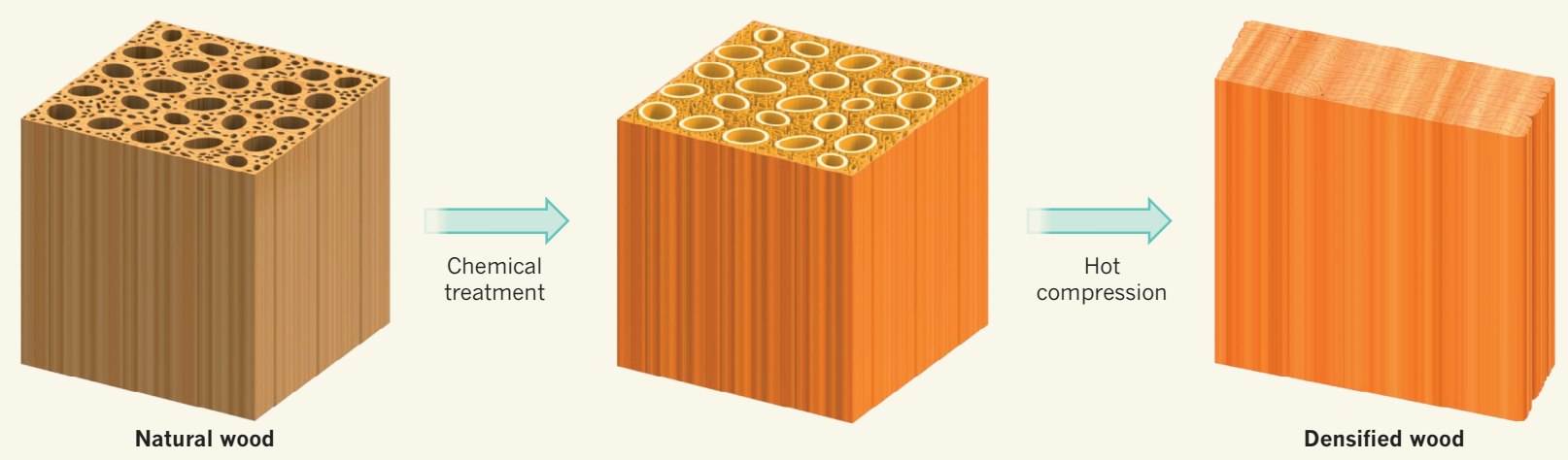

Figure $1 \mid$ A process for densifying wood. Natural wood contains pores formed from the remains of parallel, tube-like cells, the walls of which contain cellulose, along with biopolymers known as lignin and hemicelluloses. Song et al. ${ }^{2}$ treated natural wood with a mixture of sodium hydroxide and sodium sulfite, which partly removed the lignin and hemicelluloses. They then compressed the wood at about $100^{\circ} \mathrm{C}$, which caused the cells to collapse. The resulting material was about 3 times as dense as natural wood, and about 11 times as stiff and strong making it potentially useful for high-strength engineering applications. (Adapted from ref. 2.) 
compressive load along its axis is often limited by the risk of buckling; for a given column width and compressive load, higher columns can be built by using materials that have a higher Young's modulus ( $E$, a measure of stiffness). When the height of the column is not limited by an external load, but by just its own weight, then greater heights can be attained using a less dense material: the aim in this context is to maximize the ratio of $E$ to the density $\rho$, rather than just $E$. And when the goal is to build the highest possible column using a fixed mass of material, then it is best to maximize $E / \rho^{2}$. (Maintaining a constant mass is relevant to plants, because synthesizing material is a major cost for them; maximizing $E / \rho^{2}$ corresponds to the most economical way of growing the highest possible column at fixed material costs.)

A consideration of these principles reveals that Song and colleagues' densified wood should perform better than natural, porous wood in the first two scenarios (in which $E$ or $E / \rho$ need to be as large as possible), but only about equally well in the third situation, for which $E / \rho^{2}$ is maximized, on the basis of the changes in stiffness and density reported by the authors. This indicates that trees do not lose much by making wood porous, and that the introduction of pores for water transport comes at no extra material cost. Perhaps because of this, the height of trees is likely to be limited more by hydraulic constraints linked to water transport than by mechanical constraints ${ }^{8}$. Similarly, many advanced-engineering applications require materials that have high stiffness and strength, but in some cases porous materials would increase performance, rather than decrease it.

All biological materials are active, and adapt their internal structure to their function and to environmental needs. Two strategies can be used to repurpose such materials for engineering applications. One is to modify the material to comply with specifications in industrial design, as exemplified by Song et al. with their densification procedure. The other, perhaps more conventional, option is to adapt designs to the properties of natural materials. The latter approach is more sustainable, but would require greater knowledge of how structure relates to function in such materials, and the development of new design approaches ${ }^{9,10}$.

Peter Fratzl is in the Department of Biomaterials, Max Planck Institute of Colloids and Interfaces, Potsdam 14424, Germany. e-mail:fratzl@mpikg.mpg.de

1. Ramage, M. H. et al. Sustain. Energ. Rev. 68, 333-359 (2017)

2. Song, J. et al. Nature 554, 224-228 (2018).

3. Erickson, E. Mechanical Properties of Laminated Modified Wood (US Dept Agriculture, 1965)

4. Ashby, M. F. Metall. Trans. A 14, 1755-1769 (1983).

5. Ashby, M. F., Gibson, L. J.,Wegst, U. \& Olive, R. Proc. R. Soc. Lond. A 450, 123-140 (1995).

6. Gibson, L. J., Ashby, M. F., Karam, G. N., Wegst, U. \& Shercliff, H. R. Proc. R. Soc. Lond. A 450, 141-162 (1995).

7. Fratzl, P. \& Weinkamer, R. Prog. Mater. Sci. 52,
9. Schäffner, W. in +Ultra Knowledge \& Gestaltung (eds Doll, N., Bredekamp, H. \& Schäffner, W.) 23-32

(Seemann, 2017).

10. Fratzl, P. in +Ultra Knowledge \& Gestaltung (eds Doll, N. Bredekamp, H. \& Schäffner, W. 173-178 (Seemann, 2017).

\section{Many mutations in one clinical-trial basket}

\section{When abnormality in a gene is linked to cancer and a drug targets the encoded protein, how can the patients who will respond to the drug be identified if the gene is mutated in many different ways in many different cancers? SEE ARTICLE P.189}

\section{ELAINE R. MARDIS}

$\longrightarrow$ ancer usually arises from genomic abnormalities. However, the number and complexity of genetic alterations in tumours can make it difficult to predict whether, and in which tissues, a particular mutation in a specific cancer-linked gene will drive tumour growth. This poses a challenge when trying to identify effective treatments. For example, if a drug that targets a specific protein can treat a person with breast cancer who has a mutation in the gene encoding the protein, could the drug treat another patient who has a different mutation in that gene? And could it treat a person with a mutation in the same gene, but in a tumour that has developed in a different tissue? On page 189, Hyman et al. ${ }^{1}$ report the outcome of a clinical trial testing the ability of the drug neratinib, which inhibits HER2 and HER3 tyrosine kinase enzymes, to reduce or eliminate tumours. The drug was tested on 21 types of cancer in 141 people who had a total of 42 different mutations affecting one of the enzymes.

Studies in the 1970s revealed that certain chromosomal DNA aberrations can be linked to the development of specific cancer types, and that an amplification in the number of copies of particular genes can have a tumourpromoting effect ${ }^{2}$. For example, a highly lethal type of breast cancer is linked ${ }^{3}$ to amplification of the gene ERBB2 and an increase in the level of the HER2 protein that it encodes. HER2 amplification occurs in several other cancers ${ }^{4}$, including colorectal adenocarcinoma and bladder cancer. This understanding led to efforts to develop treatments to stop the action of such overexpressed proteins, resulting in several HER2-targeted therapies that are used in the clinic ${ }^{5}$ to prolong survival in people whose cancers have amplification of ERBB2. Other links between ERBB2 abnormalities and cancer have been identified;

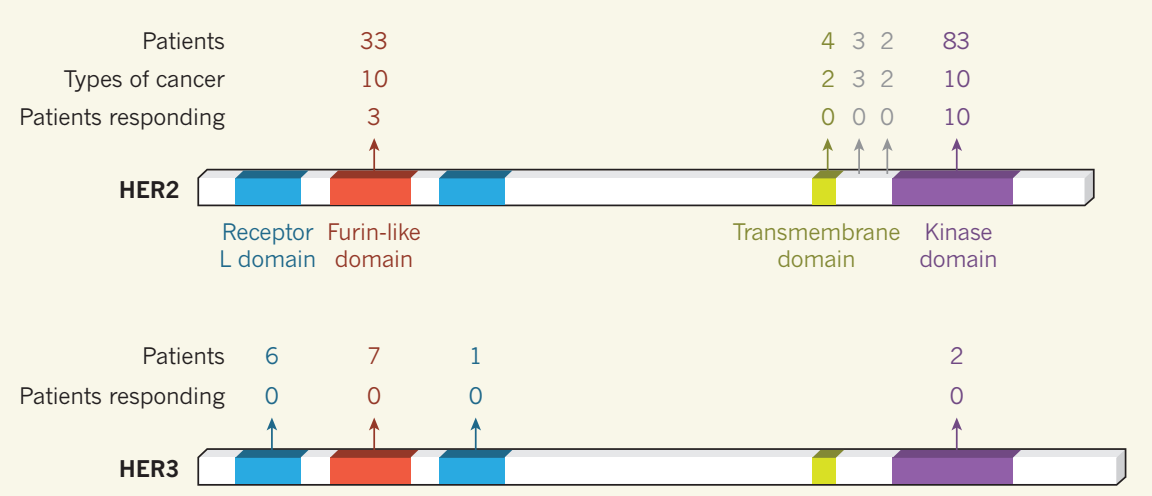

Figure 1 | Results of a cancer clinical trial. Hyman et al. ${ }^{1}$ report the outcome of a study testing how effectively the drug neratinib can treat tumours. The tyrosine kinase enzymes HER2 and HER 3 have been linked to tumour growth and can be inhibited by neratinib. The 141 patients tested had a range of mutations that altered HER2 or HER3, and, between them, had many different tumour types. The protein structures are shown, and arrows indicate the domains or interdomain locations at which protein alterations due to mutations were found. For the HER2 data shown, the cancers were grouped into ten cancer-type categories: biliary, bladder, breast, cervical, colorectal, endometrial, gastro-oesophageal, lung, ovarian or other (for all other cancer types). Responding patient numbers indicate those whose best overall response to the drug was a partial or complete response - a decrease or absence, respectively, of detectable cancer at the end of the trial. 